\title{
Phonon properties of one-dimensional nanocrystalline solids
}

\author{
X. H. Yan \\ Institute of Solid State Physics, Academia Sinica, P.O. Box 1129, Hefei 230031, People's Republic of China* \\ and Department of Physics, Xiangtan University, Xiangtan 411105, People's Republic of China \\ Lide Zhang \\ Institute of Solid State Physics, Academia Sinica, P.O. Box 1129, Hefei 230031, People's Republic of China \\ Zhuping Duan \\ Institute of Mechanics, Academia Sinica, Beijing, 100080, People's Republic of China \\ Shuzhi Cai \\ Institute of Solid State Physics, Academia Sinica, P. O. Box 1129, Hefei 230031, People's Republic of China
}

(Received 23 January 1995; revised manuscript received 19 October 1995)

\begin{abstract}
We study phonon properties of one-dimensional nanocrystalline solids that are associated with a model nanostructured sequence. A real-space renormalization-group approach, connected with a series of renormalization-group transformations, is developed to calculate numerically the local phonon Green's function at an arbitrary site, and then the phonon density of states of these kinds of nanocrystalline chains. Some interesting phonon properties of nanocrystalline chains are obtained that are in qualitative agreement with the experimental results for the optical-absorption spectra of nanostructured solids.
\end{abstract}

Research on nanometer-sized materials, including nanocrystalline solids, nanostructured semiconductors, and nanometer-sized amorphous materials, was initiated more than a decade ago. Recently, due to developments in materials processing and synthesis, it is possible to produce artificial structures with nanometer sizes whose chemical compositions and shapes can be controlled. Many types of nanostructured materials, such as the nanocrystalline solids $\mathrm{Al}_{2} \mathrm{O}_{3}$ (Ref. 1) and $\mathrm{TiO}_{2},{ }^{2}$ nanostructured semiconductors GaAs- $\mathrm{Al}_{x} \mathrm{Ga}_{1-x} \mathrm{As}$ (Ref. 3) and $\mathrm{Si}^{4}{ }^{4}$ nanometer-sized glasses $\mathrm{SiN}_{X}$ (Ref. 5) and $\mathrm{SiO}_{2}$, and so on have been successfully fabricated in the laboratory. It is found experimentally that these nanostructured materials exhibit various interesting physical properties caused by interface effects and size effects, which are significantly different from those of crystalline and glassy materials with the same chemical compositions. This may be the reason why experimental studies of nanostructured materials have been remarkably attractive. ${ }^{6}$ As for theoretical investigations of physical properties of nanostructured solids, however, difficulties arise because nanostructured solids are too small to behave like their bulk parent compounds and too large to behave like atoms or molecules. This means that it is not convenient to apply directly either molecular dynamics or crystalline dynamics to the study of their phonon spectral properties. Based on the fact that nanocrystalline solids consist of series of individual nanocrystals in which the atomic arrangements are periodic with short-range orders differing from those in crystals or glasses, here we develop a real-space renormalization-group (RG) scheme, which was introduced in Ref. 7, and then extended widely ${ }^{8}$ to the study of electronic and phonon properties of condensed matter, to study the phonon properties of nanocrystalline solids. Some interesting conclusions are obtained.

As one knows, one-dimensional problems are connected with clear physical ideas and effective mathematical methods. Due to the rich physical properties of one-dimensional nanostructured solids, such as GaAs- $\mathrm{Al}_{x} \mathrm{Ga}_{1-x} \mathrm{As}$ (Ref. 3) and $\mathrm{Si}^{4}$ we focus on a nanocrystalline chain presented sche matically in Fig. 1(a), which contains two types of individual nanocrystals $L_{1}$ and $L_{2}$ with $N_{1}$ and $N_{2}$ atoms, respectively. The nanocrystalline chain is a periodic one with the unit cell

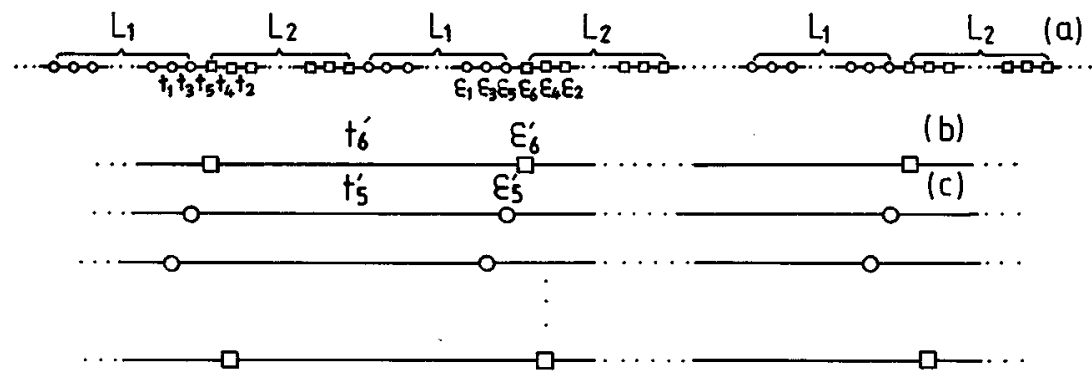

FIG. 1. Schematic representation of (a) the original nanocrystalline chain, (b) the renormalized periodic chain transferred by transformation $D_{1}^{(1)}$, and (c) the renormalized periodic chain transferred by transformation $D_{2}^{(1)}$. 
$L_{1}+L_{2}$. As far as atom configurations in an individual nanocrystal with $N$ sites are concerned, two kinds of sites can be distinguished: One is crystal sites which have periodic configurations, another is boundary sites whose periodicity is broken. Our theoretical work is mainly on phonon properties of these kinds of nanocrystalline chains, which are clearly related to their absorption spectra (AS) and luminescence spectra, satisfying the vibrational equation ${ }^{9}$

$$
\begin{gathered}
\left(\frac{K_{i, i+1}+K_{i, i-1}}{m_{i}}-\omega^{2}\right) U_{i}+\frac{K_{i, i+1}}{\sqrt{m_{i} m_{i+1}}} U_{i+1} \\
+\frac{K_{i, i-1}}{\sqrt{m_{i} m_{i-1}}} U_{i-1}=0,
\end{gathered}
$$

where $m_{i}$ and $U_{i}$ are the mass and the vibrational amplitude at site $i$, and $K_{i, i \pm 1}$ represents the spring constant coupling two nearest-neighbor sites. Depending on the local environment of site $i$, we choose the spring constants $\left\{K_{i, i \pm 1}\right\}$ to have five kinds of values $\left\{K_{i}\right\}(i=1,2, \ldots, 5)$ associated with a nanostructured sequence, while the masses $\left\{m_{i}\right\}$ take two values $M_{1}$ and $M_{2}$. For the sake of convenience, analogous to defining pseudosite parameters $\left\{t_{i, i \pm 1}\right\}$ by the five relations

$$
t_{i, i \pm 1}=\left\{\begin{array}{c}
T_{1}=K_{1} / M_{1} \\
T_{2}=K_{2} / M_{2} \\
T_{3}=K_{3} / M_{1} \\
T_{4}=K_{4} / M_{2} \\
T_{5}=K_{5} / \sqrt{M_{1} M_{2}}
\end{array}\right.
$$

we choose six other pseudosite parameters $\left\{\epsilon_{i}\right\} \quad(i$ $=1,2, \ldots, 6)$ given by

$$
\epsilon_{i}=\left\{\begin{array}{lllll}
\epsilon_{1}=2 K_{1} / M_{1} & \text { if } K_{i, i-1}=K_{i, i+1}=K_{1} & & \\
\epsilon_{2}=2 K_{2} / M_{2} & \text { if } K_{i, i-1}=K_{i, i+1}=K_{2} & & \\
\epsilon_{3}=\left(K_{1}+K_{3}\right) / M_{1} & \text { if } K_{i, i-1} \quad \text { and } K_{i, i+1} \text { are } K_{1} \text { and } K_{3} \\
\epsilon_{4}=\left(K_{2}+K_{4}\right) / M_{2} & \text { if } K_{i, i-1} \quad \text { and } K_{i, i+1} \text { are } K_{2} \text { and } K_{4} \\
\epsilon_{5}=\left(K_{3}+K_{5}\right) / M_{1} & \text { if } K_{i, i-1} \quad \text { and } K_{i, i+1} \text { are } K_{3} \text { and } K_{5} \\
\epsilon_{6}=\left(K_{4}+K_{5}\right) / M_{2} & \text { if } K_{i, i-1} \text { and } K_{i, i+1} \text { are } K_{4} \text { and } K_{5} .
\end{array}\right.
$$

In addition to $\left\{t_{i, i \pm 1}\right\}$, these pseudosite parameters $\left\{\epsilon_{i}\right\}$ (i $=1,2, \ldots, 6)$ are associated with six kinds of sites $S_{i}(i$ $=1,2, \ldots, 6)$, of which $S_{1}$ and $S_{2}$, respectively, are the crystal sites of nanocrystals $L_{1}$ and $L_{2}$, and $S_{3}, S_{4}, S_{5}$, and $S_{6}$ are the boundary sites of interfaces between nanocrystals $L_{1}$ and $L_{2}$ [see Fig. 1(a)]. Then Eq. (1) can be rewritten as

$$
\left(\omega^{2}-\epsilon_{i}\right) U_{i}=t_{i, i+1} U_{i+1}+t_{i, i-1} U_{i-1},
$$

which has the same form as a tight-binding Hamiltonian $\mathbf{H}$. Introducing Green's function ${ }^{7} G(Z)=(Z \mathbf{I}-\mathbf{H})^{-1}$ with unit matrix $\mathbf{I}$, one finds that the matrix element $G_{i j}$ satisfies

$$
\left(Z-\epsilon_{i}\right) G_{i j}=\delta_{i j}+\sum_{k} t_{i k} G_{k j}, \quad i, j=0, \pm 1, \pm 2, \ldots,
$$

where $\delta_{i j}$ is the Kronecker delta and $Z=\omega^{2}+i 0^{+}$.

In order to calculate the Green's function $\left\{G_{i i}\right\}$ at sites $\{i\}$ of nanocrystalline chains, we first use a RG transformation $D_{1}^{(1)}$ to decimate all the sites except half of the sites $S_{6}$ in the original nanocrystalline chain. The original chain is then transformed into a simple periodic renormalized chain with only sites of the kind $S_{6}$, while the site parameters $\epsilon_{6}$ and $t_{i, i \pm 1}$ are replaced by two renormalized values $e_{6}^{\prime}$ and $T_{6}^{\prime}$ [see Fig. 1(b)]. From Eq. (5), the set of RG equations of pseudosite parameters $\epsilon_{6}^{\prime}$ and $T_{6}^{\prime}$ is given by

$$
\begin{gathered}
\epsilon_{6}^{\prime}=\epsilon_{6}+\frac{T_{4}^{2}\left(V_{N_{1}-4} Z_{2}-T_{3}^{2} X_{N_{1}-4} Y_{N_{2}-4}\right)+T_{5}^{2}\left(Z_{1} R_{N_{1}-4}-T_{3}^{2} W_{N_{2}-4} P_{N_{1}-4}\right)}{Z_{1} V_{N_{1}-4}-T_{3}^{2} X_{N_{1}-4} W_{N_{2}-4}}, \\
T_{6}^{\prime}=T_{1} T_{2} T_{3}^{2} T_{4}^{2} T_{5}^{2} /\left(Z_{1} V_{N_{1}-4}-T_{3}^{2} X_{N_{1}-4} W_{N_{2}-4}\right),
\end{gathered}
$$

corresponding to the RG transformation $D_{1}^{(1)}$, where 


$$
\begin{gathered}
g=\left(Z-\epsilon_{1}\right) / 2 T_{1}, \\
h=\left(Z-\epsilon_{2}\right) / 2 T_{2}, \\
P_{N}=\left(Z-\epsilon_{3}\right) \mathscr{U}_{N}(g)-T_{1} \mathscr{U}_{N-1}(g), \\
Q_{N}=\left(Z-\epsilon_{4}\right) \mathscr{U}_{N}(h)-T_{2} \mathscr{U}_{N-1}(h), \\
R_{N}=\left(Z-\epsilon_{3}\right) P_{N}-T_{1} P_{N-1}, \\
S_{N}=\left(Z-\epsilon_{4}\right) Q_{N}-T_{2} Q_{N-1}, \\
X_{N}=\left(Z-\epsilon_{5}\right) P_{N}-T_{3}^{2} \mathscr{C}_{N}(g), \\
Y_{N}=\left(Z-\epsilon_{6}\right) Q_{N}-T_{4}^{2} \mathscr{U}_{N}(h), \\
V_{N}=\left(Z-\epsilon_{5}\right) R_{N}-T_{3}^{2} P_{N}, \\
W_{N}=\left(Z-\epsilon_{6}\right) S_{N}-T_{4}^{2} Q_{N}, \\
Z_{1}=\left(Z-\epsilon_{5}\right) W_{N_{2}-4}-T_{5}^{2} S_{N_{2}-4}, \\
Z_{2}=\left(Z-\epsilon_{5}\right) Y_{N_{2}-4}-T_{5}^{2} Q_{N_{2}-4},
\end{gathered}
$$

and $\mathscr{C b}_{N}(X)$ is the $N$ th Chebyshev polynomial of the second kind, which satisfies the recursion relation

$$
\mathscr{C b}_{N}(X)=2 X \mathscr{U}_{N-1}(X)-\mathscr{C b}_{N-2}(X)
$$

with the initial conditions $\mathscr{U}_{-2}(X)=-1$ and $\mathscr{C}_{-1}(X)=0$. Correspondingly, by introducing other RG transformations $D_{j}^{(1)}\left(j=2,3, \ldots\right.$, and $\left.N_{1}+N_{2}\right)$ based on the RG scheme, one can also transform the original nanocrystalline chain into other simple periodic renormalized chains in each of which half of the sites $S_{i}(i=1$, or $2, \ldots, 5)$ in the original chain with renormalized site parameters $\epsilon_{i}^{\prime}$ and $T_{i}^{\prime}$, remain, while all other kinds of sites and half of the sites $S_{i}$ in the original chain are decimated. According to the geometric properties of the presented RG transformation $D_{j}^{(1)}$, the corresponding sets of RG equations can be obtained from Eq. (5). For instance, we can use a RG transformation $D_{2}^{(1)}$ to transfer the original nanocrystalline chain to a renormalized simple periodic chain with only the kind of sites $S_{5}$ s [see Fig. 1(c)]. From Eq. (5), the set of RG equations corresponding to $D_{2}^{(1)}$ is given by

$\epsilon_{5}^{\prime}=\epsilon_{5}+\frac{T_{3}^{2}\left(Z_{1} X_{N_{1}-4}-T_{5}^{2} P_{N_{1}-4} W_{N_{2}-4}\right)+T_{5}^{2}\left(V_{N_{1}-4} Z_{2}-T_{5}^{2} R_{N_{1}-4} S_{N_{2}-5}\right)}{Z_{1} V_{N_{1}-4}-T_{5}^{2} R_{N_{1}-4} W_{N_{2}-4}}$,

$T_{5}^{\prime}=T_{1} T_{2} T_{3}^{2} T_{4}^{2} T_{5}^{2} /\left(Z_{1} V_{N_{1}-4}-T_{5}^{2} R_{N_{1}-4} W_{N_{2}-4}\right)$,

where $g, h, P_{N}, Q_{N}, R_{N}, S_{N}, X_{N}, Y_{N}, V_{N}$, and $W_{N}$ are given by relations (7), and

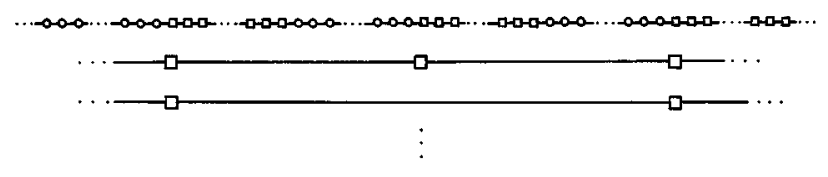

FIG. 2. A schematic representation of the RG procedure for a nanocrystalline chain, in which half of the sites $S_{6}$ remain.

$$
\begin{aligned}
& Z_{1}=\left(Z-\epsilon_{6}\right) W_{N_{2}-4}-T_{4}^{2} Y_{N_{2}-4}, \\
& Z_{2}=\left(Z-\epsilon_{6}\right) S_{N_{2}-4}-T_{4}^{2} Q_{N_{2}-4} .
\end{aligned}
$$

As the original chain is reduced to a simple periodic chain with only one kind of site $S_{i}$, one can easily calculate its physical properties in many ways. Here we present a further RG transformation $D^{(2)}$ in order to decimate the renormalized periodic chain successively. Each procedure removes half of the sites in the periodic chain (see Fig. 2). Assuming the pseudosite parameters are symboled by $\epsilon$ and $T$, we can also obtain, from Eq. (5), the set of RG equations

$$
\epsilon^{\prime}=\epsilon+2 T^{2} /(Z-\epsilon), \quad T^{\prime}=T^{2} /(Z-\epsilon),
$$

corresponding to the RG transformation $D^{(2)}$, where $\epsilon^{\prime}$ and $T^{\prime}$ are the renormalized parameters of the pseudosites in the renormalized subchain.

After infinite iterations of above transformations, some of a certain kind of sites in the original chain are always preserved with renormalized pseudosite parameters. Applying suitable combinations of the RG transformations presented above, a given site in the original chain should remain in a certain renormalized subchain. It is found that the final re-
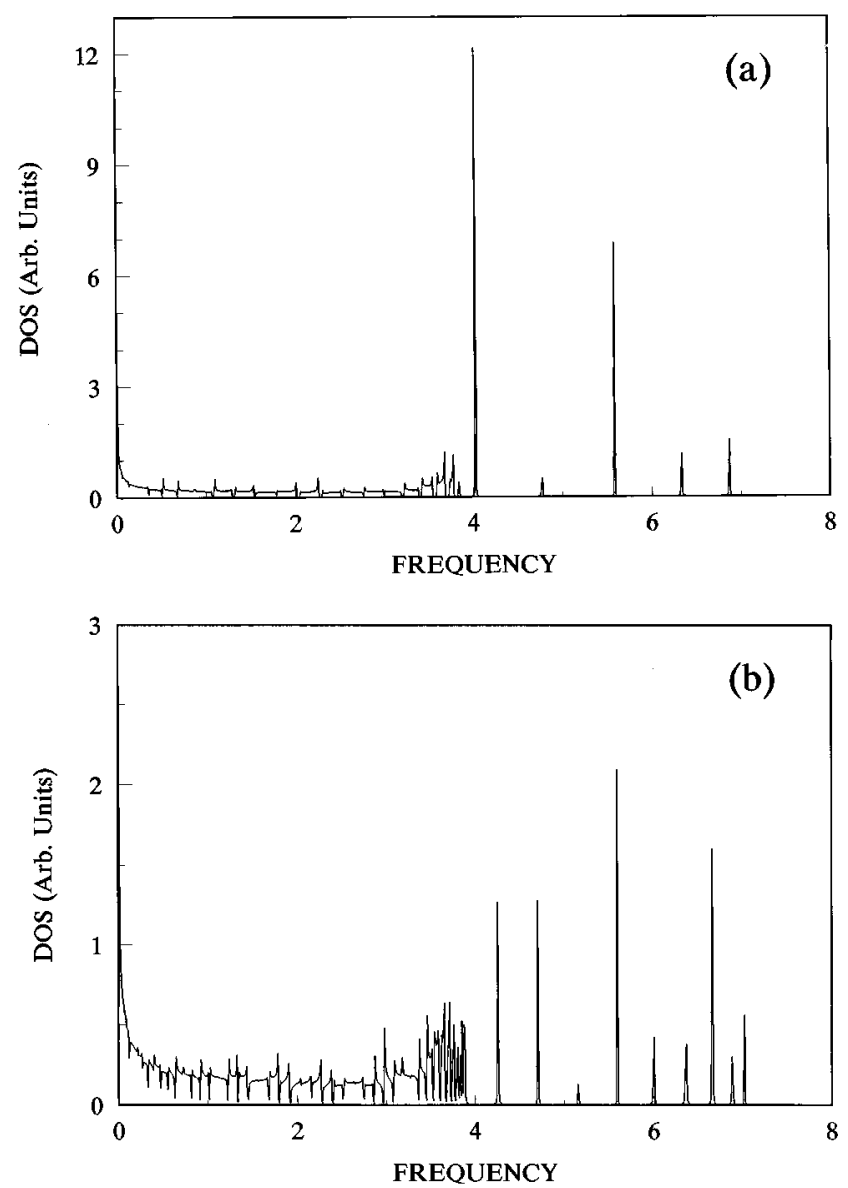

FIG. 3. The phonon DOS (arbitrary units) of nanocrystalline chains with site parameters $K_{1}=180, K_{2}=140, K_{3}=170, K_{4}=150$, $K_{5}=160, M_{1}=102$, and $M_{2}=145$, in which the frequency coordinate represents $\omega^{2}$ (in units of $K_{1} / 180$ ) and $N_{1} / N_{2}=$ (a) $10 / 20$ and (b) $20 / 40$. 

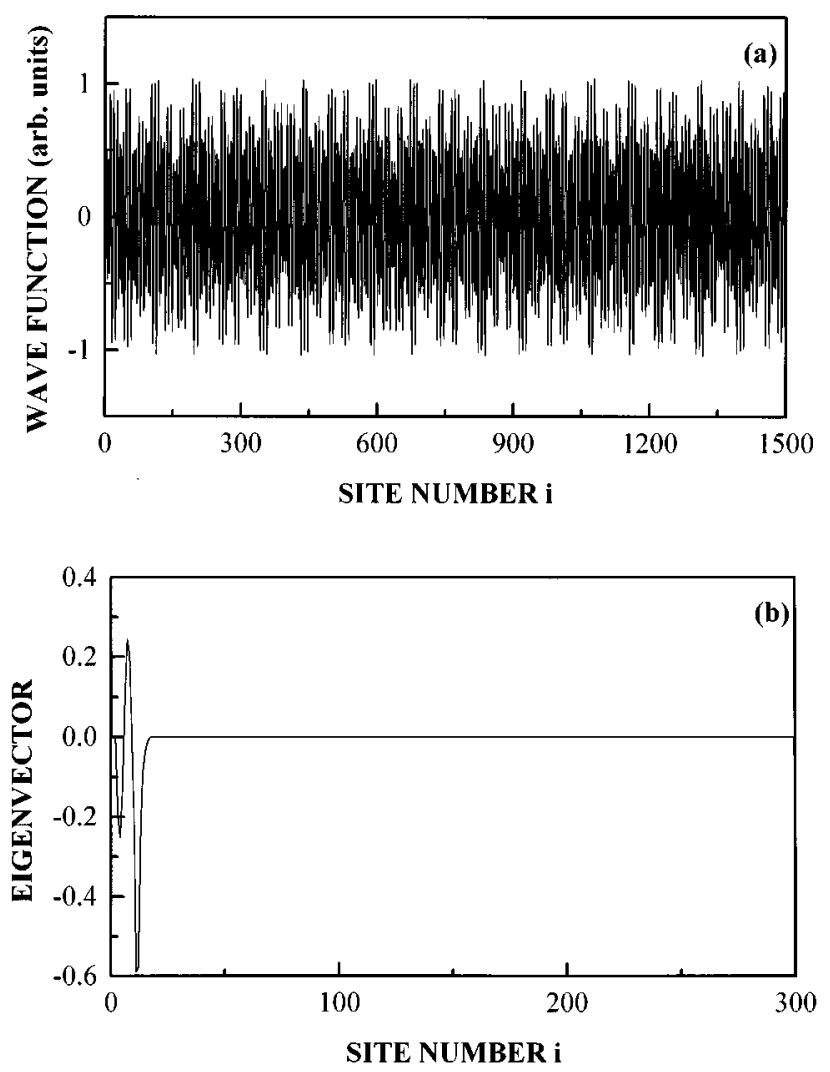

FIG. 4. (a) The wave function at the eigenfrequency $\omega^{2}=3.12018437$ of the nanocrystalline chain with $N_{1} / N_{2}=10 / 20$, of which the total number of sites is 1500 and the site parameters $K_{i}$ $\left(i=1,2,3,4\right.$, and 5) and $M_{j}(j=1$ and 2$)$ are the same as those in Fig. 3. (b) The eigenvector structure at the eigenfrequency $\omega^{2}=5.752142151$ of the nanocrystalline chain with $N_{1} / N_{2}=10 / 20$, of which the total number of sites is 300 and the site parameters $K_{i}$ $(i=1,2,3,4$, and 5$)$ and $M_{j}(j=1$ and 2$)$ are same as those in Fig. 3.

maining sites are almost isolated, because the pseudosite parameter $T_{i}$ converges to zero after successive iterations of RG procedures. Thus, from Eq. (5), the local Green's function at site $i$ satisfies

$$
G_{i i}(Z)=1 /\left(Z-\epsilon_{i}^{*}\right)
$$

where $\epsilon_{i}^{*}$ is the final value of the pseudosite parameter. So we can calculate many physical properties of these kinds of nanocrystalline chains, which are associated with the Green's function. For example, in order to compare with experimental results on spectral properties of AS and luminescence of nanostructured solids, we calculate numerically the local phonon density of states (LDOS) at certain sites, and the density of states (DOS) of the nanocrystalline chains, which are given by

$$
\begin{gathered}
\rho_{i}\left(\omega^{2}\right)=-\frac{1}{\pi} \operatorname{Im} G_{i i}\left(\omega^{2}+i 0^{+}\right), \\
\rho\left(\omega^{2}\right)=-\frac{1}{N \pi} \operatorname{Im}\left[\sum_{i} G_{i i}\left(\omega^{2}+i 0^{+}\right)\right],
\end{gathered}
$$
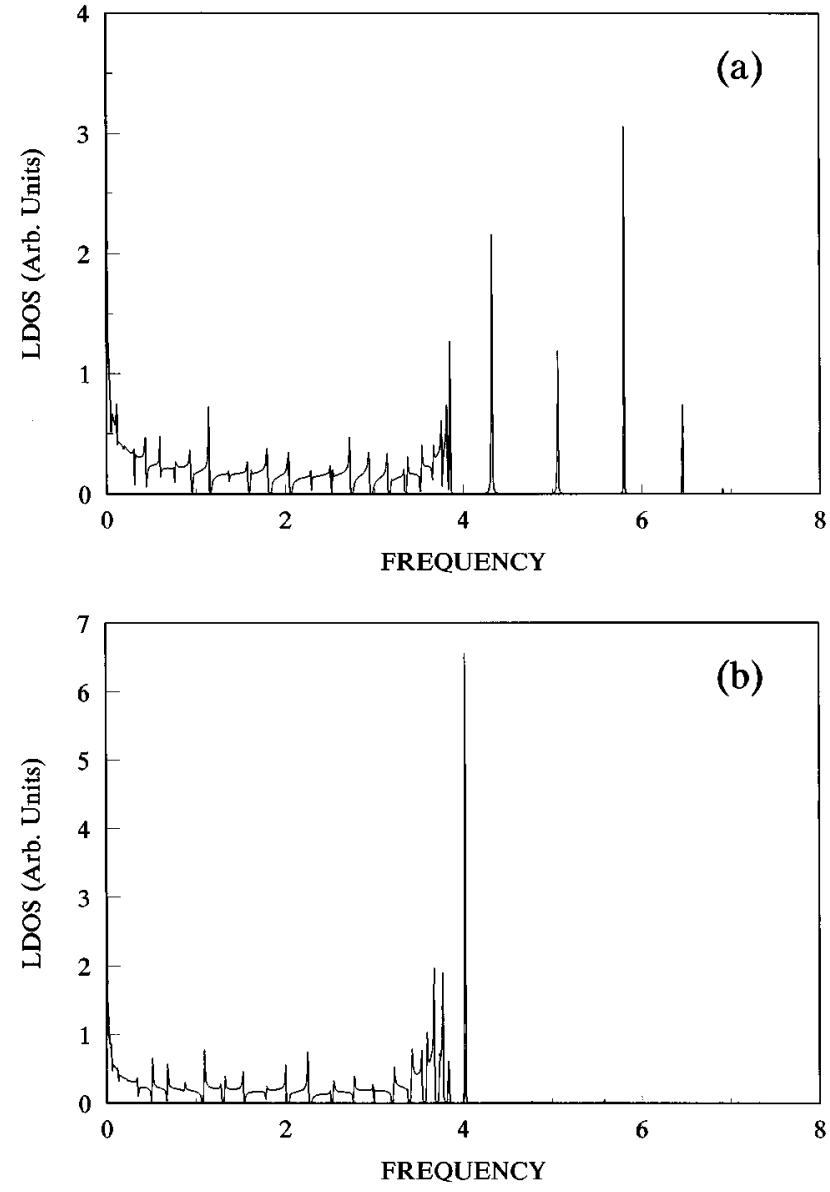

FIG. 5. The phonon LDOS (arbitrary units) at two different sites of nanocrystalline chain with $N_{1} / N_{2}=10 / 20$, in which the frequency coordinate represents $\omega^{2}$, and the site parameters $K_{i}(i=1$, $2,3,4$, and 5) and $M_{j}(j=1$ and 2) are chosen to be the same as those in Fig. 3. (a) The nearest boundary site $S_{6}$. (b) The crystal site $S_{2}$.

where Im and $N$ denote, respectively, the imaginary part of a complex quantity and the number of sites in the studied nanocrystalline chain. Comparing a $\mathrm{GaAs}-\mathrm{Al}_{x} \mathrm{Ga}_{1-x} \mathrm{As}$ nanostructured solid ${ }^{3}$ to the kind of nanocrystalline chain studied here, we choose the site parameters to be $K_{1}=180$, $K_{2}=140, K_{3}=170, K_{4}=150, K_{5}=160$ (arbitrary units), $M_{1}=102$, and $M_{2}=145$ (arbitrary units). Two types of nanocrystalline chains, with $N_{1} / N_{2}=10 / 20$ and 20/30 are studied.

Figures 3(a) and 3(b) show the DOS of the two nanocrystalline chains with sizes $N_{1} / N_{2}=10 / 20$ and 20/40, respectively. It is obvious that the phonon spectrum of nanocrystalline chains is divided into high- and low-frequency regions between which there is a gap. In the low-frequency region, the LDOS exhibits smooth structures. This indicates that the spectrum in the low-frequency region is generally extended. Similar conclusion can be reached from Fig. 4(a), which shows the wave function at the eigenfrequency $\omega^{2}=3.12018437$ of a nanocrystalline chain with $N_{1} / N_{2}=10 / 20$, and with periodic boundary conditions, where the number of sites is 1500 . In the high-frequency region, however, multiple sharp peaks appear. Using the TQLI (tridiagonal QL Implicit) routine, we show, in Fig. 
4(b), the eigenvector structure of the nanocrystalline chain with $N_{1} / N_{2}=10 / 20$, where $\omega^{2}=5.752142151$. Owing to the limitation of the capacity of our computer, here we consider a system with 300 sites, and stationary boundary conditions. One can see, from Fig. 4(b) as well as from Figs. 3(a) and $3(b)$, that the sharp peaks correspond to the localized states which are confined within a very small region. This kind of localized states is similar to the confined states found in twodimensional quasicrystals, ${ }^{10}$ which are different from the extended states in crystals, the critical states in quasiperiodic systems, or the localized states in amorphous materials. Additionally, the positions of the highest frequency sharp peaksare shifted to higher frequencies as the numbers $N_{1}$ and $N_{2}$ decrease. In terms of the optical spectroscopy theory, the infrared AS coefficient $\alpha(\omega)$ of matter is directly proportional to the DOS: ${ }^{11}$

$$
\alpha(\omega) \propto|M(\omega)|^{2} \rho^{\prime}(\omega),
$$

where $\rho^{\prime}(\omega)=2 \omega \rho\left(\omega^{2}\right)$ and the weight integral $|M(\omega)|^{2}$ of the dipole transition is a continuous function of the frequency $\omega$. These facts imply that the infrared AS of one-dimensional nanocrystalline solids are shifted to higher frequencies, i.e., are blue-shift phenomena, as the sizes of individual nanocrystals decrease. This conclusion is identical to the experimental results ${ }^{4}$ on nanostructured solids. Another interesting feature in the phonon DOS of nanocrystalline chains is that the number of sharp peaks decreases as the sizes of individual nanocrystals decrease. It is interesting that similar phenomena are found in experiments on $\mathrm{SiO}_{2} \cdot{ }^{12}$ Figures 5(a) and 5(b) display the phonon LDOS at a boundary site $S_{6}$ and a crystal site $S_{2}$ of a nanocrystalline chain. Similar conclusions to those reached above can be found. Furthermore, it can be seen from Figs. 5(a) and 5(b) that, in the highfrequency region, there are fewer sharp peaks in the LDOS's of the crystal sites than in the LDOS's of the boundary site. This observation implies that the sharp peaks in the highfrequency region are due mainly to the boundary sites in the nanocrystalline chain.

In summary, we have constructed a chain model of nanocrystalline solids. By applying a real-space renormalizationgroup scheme, physical properties as well as the local Green's function at any given site of the nanocrystalline chains can be calculated. As typical examples, the phonon DOS and LDOS at several sites have been calculated numerically. In the low-frequency region, nanocrystalline chains generally possess extended states. In the highfrequency region, however, the phonon DOS of the nanocrystalline chains exhibit multiple sharp peaks corresponding to localized states due to strong interface effects. As the sizes of the individual nanocrystalline grains decrease, the positions of the highest frequency sharp peaks shift to the higher frequency side, while the number of peaks at the highfrequency region decreases. This shows that there exist size effects in the nanostructured chains.

We thank J. Q. You for useful discussion. This work was supported by a key project of the National Climbing Program Research of China on fundamental research.
*Mailing address.

${ }^{1}$ R. Birringer, H. Gleiter, H. P. Klein, and P. Marquardt, Phys. Lett. A 102, 365 (1984); C. Mo, Z. Yuan, L. Zhang, and C. Xie, Nanostruct. Mater. 2, 47 (1993).

${ }^{2}$ C. A. Melendres et al., J. Mater. Res. 4, 1246 (1989); J. C. Parker, Appl. Phys. Lett. 57, 27 (1990).

${ }^{3}$ D. S. Chemla, Phys. Today 46 (6), 22 (1993).

${ }^{4}$ Y. Kaneitsu et al., Phys. Rev. B 46, 3916 (1992); H. Takagi et al., Appl. Phys. Lett. 56, 2379 (1990).

${ }^{5}$ T. Goto and T. Hirai, J. Mater. Sci. 24, 821 (1989); Chimei Mo, Lide Zhang, Chunyi Xie, and Tao Wang, J. Appl. Phys. 73, 5185 (1993).

${ }^{6}$ As a fact, an academic journal Nanostructured Materials in this special field started publication in 1992.

${ }^{7}$ B. W. Southern, A. A. Kumar, P. D. Loly, and A. M. S. Trembley, Phys. Rev. B 27, 1405 (1983); M. O. Robbins and B. Koiller, ibid. 27, 7703 (1983); Youyan Liu, R. Riklund, and K. A. Chao, J. Phys. C 17, L843 (1984).

${ }^{8}$ J. P. Lu, T. Odagaki, and J. L. Birman, Phys. Rev. B 33, 4809 (1986); M. Kantha and R. B. Stincombe, J. Phys. A 20, 495 (1987); X. H. Yan, J. X. Zhong, J. R. Yan, and J. Q. You, Phys. Rev. B 46, 6071 (1992), and references therein.

${ }^{9}$ For a review, see G. K. Horton and A. A. Maradudin, Dynamical Properties of Solids (North-Holland, Amsterdam, 1974), Vols. 1 and 2; Dynamical Properties of Solids (North-Holland, Amsterdam, 1980), Vol. 3.

${ }^{10}$ M. Arai, T. Tokihiro, T. Fujiwara, and M. Kohmoto, Phys. Rev. B 38, 1621 (1988).

${ }^{11}$ R. C. Fang, L. Ley, H. R. Shanks, K. J. Gruntz, and M. Cardona, Phys. Rev. B 22, 6140 (1980), and references therein.

${ }^{12}$ B. Zhang et al. (unpublished). 